\title{
Untersuchungen (mit Hilfe der Weichardtschen Reaktion) über die Beeinflussung der Katalysatorentätigkeit des Blutes und von Gewebeflüssigkeiten im Kindesalter.
}

\author{
Von \\ Dr. Ernst Stettner, \\ Assistenzarzt. \\ (Aus der Universitäts-Kinderklinik, Erlangen.) \\ Mit 7 Textfiguren. \\ (Eingegangen am 18. September 1913.)
}

Vor kurzem hat Weichardt mit seinen Schülern eine Methode bekanntgegeben, welche es gestattet, Eiweißspaltprodukte unbekannter Herkunft nachzuweisen. Es fand sich, daß organische Katalysatoren, z. B. der nach einem bestimmten Verfahren dargestellte Blutkatalysator, sowie auch anorganische Katalysatoren, wie kolloides Osmium, in ganz bestimmter Weise durch derartige Spaltprodukte beeinflußt werden. Wie unterdessen von anderen Autoren bestätigt, gilt das Gesetz, daß diese Katalysatoren durch kleine Dosen von Eiweißabbauprodukten angeregt, durch große Dosen in ihrer Wirksamkeit gehemmt werden. Es ist somit eine Methode gefunden, auf indirekte Weise, durch die Beeinflussung von Katalysatoren das Vorhandensein von Eiweißspaltprodukten zu erkennen.

Die Ausführung dieses Nachweises lehnt sich zweckmäßig an die von Chodat und $\mathrm{Bach}$ angegebene Jodkalistärkemethode an, weil man in dieser die quantitativ exakte, titrimetrische Jodbestimmung benützen kann. $5 \mathrm{ccm}$ eines Gemisches von 1,2 g löslicher Stärke und 2,0 g Jodkali in $1000 \mathrm{ccm}$ destilliertem Wasser gelöst, werden mit $2 \mathrm{ccm}$ Terpentinölwassers vereinigt, durch Abgabe von Sauerstoff aus dem an Peroxyden reichen Terpentinölwasser, wird die Bildung von blauer Jodstärke erreicht. Nach Einwirken von $1 / 2$ Stunde Dauer wird mit $1 / 1000$ n-Natriumthiosulfatlösung bis zur Farblosigkeit titriert, man benötigt hierzu meist 2-3 ccm. Der Verbrauch an Natriumthiosulfat gibt das Maß für die in $1 / 2$ Stunde gebildete Jodstärke. Der Blutkatalysator wird nach Weichardt folgendermaßen dargestellt: etwa $10 \mathrm{ccm}$ Venenblut werden von Serum befreit, der Blutkuchen mehrmals mit frischer steriler physiologischer $\mathrm{NaCl}-\mathrm{Lösung}$ gewaschen und dann die Blutkörperchen in frisch destilliertem Wasser aufgelöst. Diese Blutlösung wird in flache Petrischalen ausgegossen und im Faust-Heimschen Apparat oder mit Hilfe einer „Föhn“-Luftdouche getrocknet, die Temperatur soll 
$37^{\circ}$ nie mals übers teigen. Jetzt wird der getrocknete Blutkatalysator mit einem Spachtel zusammengeschabt und davon $0,1 \mathrm{~g}$ in $50 \mathrm{ccm}$ frisch destillierten Wassers gelöst, es empfiehlt sich anfänglich das $\mathrm{H}_{2} \mathrm{O}$ tröpfchenweise zuzusetzen und mit dem Trockenblut in einem Schälchen zu verreiben. Der trockene Blutkatalysator erhält längere Zeit seine Wirksamkeit, doch empfiehlt es sich, ihn immer frisch zu verwenden, zumal er sich in älterem Zustande schlechter auflöst; eine völlige Lösung ist dann nur nach Zusatz von einigen Tropfen $1 / 10 \mathrm{n}-\mathrm{NaHO}$ zu erreichen. Für die vorliegenden Untersuchungen ist fernerhin häufig noch kolloidales Osmium als Katalysator verwandt, dasselbe liefert wie alle anderen notwendigen Substanzen die Firma Grübler \& Co., Leipzig; $\mathrm{zu}$ unseren Versuchen war es so eingestellt, daß $3 \mathrm{ccm}$ mit $5 \mathrm{ccm}$ Jodkalistärkelösung und $2 \mathrm{ccm}$ Terpentinölwasser nach $1 / 2$ stündiger Einwirkung durch etwa $6 \mathrm{ccm}$ Natriumthiosulfatlösung wieder farblos wurden.

An Stelle des Terpentinölwassers könnte man natürlich eine Reihe anderer sauerstoffreicher Substanzen setzen, alle diese Versuche führten jedoch stets zu dem Ergebnis, daß das Terpentinölwasser allen Ersatzmitteln überlegen ist, weil es unbeeinflußt nur wenig Sauerstoff abgibt, bei Einwirken von Katalysatoren jedoch sehr reichlich, so daß bei der Titration immer greße Ausschläge vorhanden sind. Ein gewisser Nachteil besteht allerdings darin, daß das $\mathbf{O}$-Abgabevermögen des Terpentinölwassers nicht jeden Tag das gleiche ist, es können die gefundenen Werte demnach nur innerhalb einer Versuchsreihe verglichen werden.

Nach den Untersuchungen von Weichardt läßt sich mit Hilfe obenbeschriebener Methode nachweisen, daß ,,bei verschiedenen im Tierversuch erzeugten akuten Proteotoxikosen und bei ebensolchen von Menschen, bei denen die parenterale Verdauung von Eiweiß eine Rolle spielt, die Katalysatorentätigkeit des Hämoglobins gegenüber dem Normalen meist angeregt ist". Seine Untersuchungen erstrecken sich in der Hauptsache auf anaphylaktische Erscheinungen, auf den Körperzustand nach Verbrennungen und in der Schwangerschaft. Auch Engelhorn berichtet, da $\beta$ in der Schwangerschaft der Blutkatalysator im Vergleich zu dem bei dem bei Nichtschwangeren meist angeregt ist.

Das Material, welches mir zur Verfügung stand, beschränkte in gewissem Sinne meine Fragestellung. Es wurde zunächst einigen Säuglingen Blut aus der Vene entnommen und in entsprechender Weise gewaschen und getrocknet. Verschiedene Verdauungsstörungen des Säuglingsalters zeigten in dem Verhalten des Blutkatalysators anscheinend ungesetzmäßige Schwankungen. Es lag in der Natur der Versuchsanordnung, daß wir hierüber keine großen Versuchsreihen anstellen konnten, denn einem an sich geschwächten Säugling entnimmt man nur ungern wiederholt das wertvolle Blut. In 3 Fällen von Spasmophilie 
und latenter Tetanie fand sich der Blutkatalysator dem normalen gegenüber konstant erniedrigt. Folgende Zahlen belehren darüber ohne weiteres.

\begin{tabular}{c|c|c|c}
\hline $\begin{array}{c}\text { Normalblut } \\
\text { Z. }\end{array}$ & $\begin{array}{c}\text { Spasmophilie } \\
\text { Pf. }\end{array}$ & $\begin{array}{c}\text { = } \\
\text { L. }\end{array}$ & $\begin{array}{c}\text { Lat. Tetanie } \\
\text { s. }\end{array}$ \\
\hline 3,46 & 2,92 & 2,98 & $\mathbf{3 , 1 6}$
\end{tabular}

Die Ausschläge, die sich hier zeigen, sind weit außerhalb der Fehlergrenze. Die Werte entsprachen etwa dem klinischen Bilde, indem Pf., dessen Blutkatalysator am stärksten gelähmt war, das schwerste Krankheitsbild bot. Es ist selbstverständlich, daß aus diesen 3 Fällen keine bindenden Schlüsse gezogen werden können. Versuche, die eine Konstanz dieser Erscheinung beweisen sollen, müssen solange aufgeschoben werden, bis die Wintermonate wieder die entsprechenden Fälle liefern. Das Wesen der Spasmophilie ist ja bei weitem noch nicht geklärt, obenerwähnter Befund könnte die Basis zur Deutung der Versuche von Freudenberg und Klocmann abgeben, welche beim Studium des Spasmophilieproblems die Oxydationsprozesse erniedrigt fanden. Man könnte annehmen, daß eben durch den Mangel oder durch die Schädigung der Blutkatalysatoren die Oxydationsprozesse herabgesetzt würden.

Wenn auch nach unseren Versuchen Beziehungen der Spasmophilie zur Anaphylaxie unwahrscheinlich waren, da ja im anaphylaktischen Zustand meist eine Anregung des Blutkatalysators vorhanden ist, so war es doch von besonderer Wichtigkeit, besonders da in neuerer Zeit manche Ernährungsstörungen der Säuglinge als anaphylaktische Reaktion gegen Kuhmilch gedeutet werden, zu wissen, wie sich verschiedene Milcheiweißkörper gegenüber dem Blutkatalysator ïberempfindlicher Tiere verhalten.

Für den spasmophilen Zustand ist wegen ihres deletären Finflusses die Kuhmilchmolke von besonderer Bedeutung. Die Kuhmilchmolke wurde in bekannter Weise durch Pegnin gewonnen. Um für spätere Versuche ein möglichst konstantes Präparat zu besitzen, trockneten wir den nach Ätherextraktion gewonnenen Rückstand der Molke und gewannen nach Verreiben ein feinkörniges Pulver, welches sich selbst nach Zusatz von etwas N-Sodalösung schwer in Wasser löste. In gleicher Weise verfuhren wir mit dem Casein. Am 1. III. 1913 wurden eine Reihe von jungen Meerschweinchen mit je $0,1 \mathrm{~g}$ auf $5 \mathrm{ccm} \mathrm{H}_{2} \mathrm{O}$ des so gewonnenen Molkeneiweißes und Caseins subcutan sensibilisiert. Die Reinjektion versuchten wir anfangs mit den gleichen Lösungen und bekamen damit schöne anaphylaktische Zustände. Später entschlossen wir uns, reine Kuhmilchmolke und gut gewaschene Caseinaufschwemmung (Pegninmilch) zur intravenösen Reinjektion zu verwenden, da wegen der schlechten Löslichkeit unserer Trockenpräparate durch Einwirkung cor- 
pusculärer Elemente leicht Fehler hätten entstehen können. Allerdinge mußten wir jetzt einen weiteren Gesichtspunkt berücksichtigen. Molke, sowohl wie Caseinaufschwemmung sind selbst starke Katalysatoren, wie es für Kuhmilchmolke aus nebenstehender Kurve ersichtlich ist.

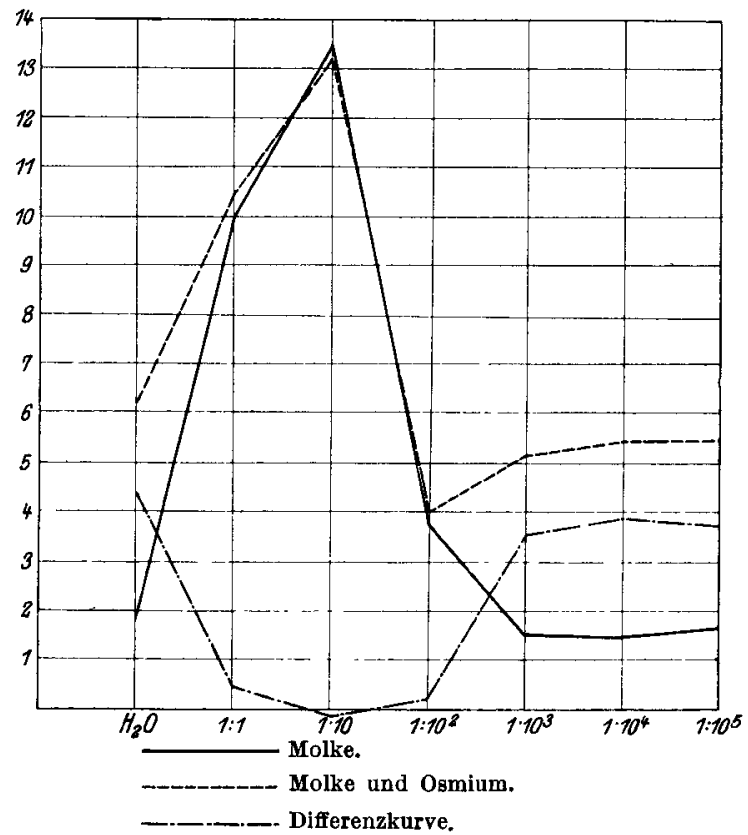

Fig. 1.

Durch die intravenöse Injektion wurde das Blut unserer Tiere mit reichlich Katalysatoren überschwemmt, was sich bei der Titration des Blutkatalysators deutlich bemerkbar machte, es mußten demnach stets das Blut eines sensibilisierten und reinjizierten Tieres, das Blut eines Tieres, dem nur kurz vor der Tötung in gleicher Weise Molke oder Casein injiziert war und das Blut eines unbehandelten normalen Meerschweinchens berücksichtigt werden. Im folgenden einige Typen:

Meerschweinchen 63. O Gew. $265 \mathrm{~g}$. Am 1. III. 13 mit 0,1: 5,0 Trockenmolkeneiweiß sensibilisiert, am 3. IV. $13 \mathrm{mit} 1 \mathrm{ccm}$ frisch bereiteter Kuhmilchmolke reinjiziert, nach 1/2 Minute typischer anaphylaktischer Shock, rascher Verfall. Sofortige Entblutung nach Eröffnung des Thorax nach Herausnahme einer enorm geblähten Lungenhälfte. Verarbeitung des Blutes in oben beschriebener Weise.

Kontrollmeerschweinchen 63a. $\sigma^{7}$ Gew. $346 \mathrm{~g}$, unvorbehandelt, bekommt in gleicher Weise $1 \mathrm{ccm}$ Molke intravenös, keine Krämpfe, Entblutung nach $12 \mathrm{Min}$. aus den Lungengefäßen.

\begin{tabular}{c|c|c}
\hline Normal-M. & M. 68 a & M. 63 . \\
\hline 3,44 & 3,60 & 3,77
\end{tabular}

Prüfung des Blutkatalysators am 4. IV. 13. 
Bei dem (anaphylaktischen) Tiere 63 wurde also durch den anaphylaktischen Shock, d. h. durch die akute Einwirkung der beim raschen Abbau des Molkeneiweißes entstandenen Eiweißspaltprodukte eine Anregung des Blutkatalysators erzielt.

Meerschweinchen 56. $\sigma^{7}, 335 \mathrm{~g}$ Gew. Am 1. III. Sensibilisierung durch subcutane Einverleibung von $0,1: 5,0$ Trockencasein, am 8. IV. intravenöse Reinjektion von $0,5 \mathrm{com}$ frisch bereiteter Caseinaufschwemmung. Nach $1 / 2$ Minute typische anaphylaktische Krämpfe, Temperatur sinkt von 36,8 auf 36,0. Entblutung wie oben, kolossale Lungenblähung.

Kontrollmeerschweinchen 56a. O, Gew. $320 \mathrm{~g}$, unvorbehandelt, erhält am gleichen Tage $0,5 \mathrm{ccm}$ Caseinaufschwemmung wie oben intravenös. Entblutung aus den Lungengefäßen nach 25 Minuten, keine Lungenblähung.

Prüfung des Blutkatalysators am 10. IV. 13.

$==\frac{\text { Normal-M. }}{4,15}=\frac{\text { M. 56a }}{4,31}=\frac{\text { M. 66 }}{4,23}==$

Für diesen Fall wäre eine mäßige Hemmung der Katalysatorentätigkeit zu verzeichnen. Sie erklärt sich folgendermaßen: Nach dem vorher schon gefundenen Gesetze, daß geringe Mengen von Eiweißspaltprodukten, wenn sie auf den Blutkatalysator nur kurze Zeit einwirken, eine Steigerung des organischen Katalysators bedingen, während größere Mengen und längere Einwirkungszeit Lähmung zu bedingen pflegen, ist es wohl denkbar, daß wir auch bei gewissen Stadien der Anaphylaxie nur geringe Anregungswerte, ja sogar Lähmung beobachten, denn es sind drei Faktoren, von denen unser Katalysator abhängig ist: 1. die Zeit der Einwirkung, 2. die Quantität, und 3. die Qualität der freiwerdenden Eiweißspaltprodukte.

Dieses inkonstante Verhalten der Reaktion je nach dem Grade der Anaphylaxie macht sie zur Untersuchung des Problemes, ob Spasmophilie und Anaphylaxie in Beziehungen stehen, ungeeignet. Nach unseren vorliegenden Untersuchungen können wir also darüber keine Entscheidung treffen.

Wie früher schon angedeutet, ist die Entnahme von mehreren Kubikzentimetern Blut bei Säuglingen eine mißliche Sache, wenn sie öfters wiederholt werden muß. Ich war daher bestrebt, die Blutentnahme zur Katalysatorenprüfung auf ein Minimum einzuschränken und entschloß mich, das Blut, wie es durch Einstich aus der Fingerbeere quillt, zur Reaktion zu verwenden. Mit einer Pipette, welche auf $20 \mathrm{cmm}$ und $2 \mathrm{ccm}$ geeicht ist, stellte ich mit Hilfe frisch destillierten Wassers eine Blutverdünnung 1 : 100 her und prüfte diese auf ihre katalysatorischen Fähigkeiten in einer größeren Versuchsreihe. 
224 E. Stettner: Untersuchungen uber die Beeinflussung der Katalysatoren-

\begin{tabular}{|c|c|c|c|c|c|}
\hline Datum & Name & Alter & $\begin{array}{l}\text { Kataly- } \\
\text { satoren- } \\
\text { wert }\end{array}$ & $\mathbf{H} \mathrm{b} \%$ & Diagnose \\
\hline 13. 5. 13 & $2 \mathrm{ccm} \mathrm{H}_{2} \mathrm{O}$ & - & 3,10 & - & - \\
\hline 1. & F. D. & 4 Mt. & 4,09 & 83 & Handphlegmone, Atrophie. \\
\hline 2. & A. $\mathrm{H}$. & $1 \mathrm{~J}$. & 3,76 & - & Pemphigus rec. \\
\hline 3. & E. Sp. & $5^{1} / 2$ Mt. & 3,71 & 81 & Ammenkind (ständig Leukocytose) \\
\hline 4. & J. H. & 1 Mt. & 4,19 & 115 & Künstliche Ernährung. \\
\hline 5. & I. L. & 8 Mt. & 4,06 & 77,5 & Decompositon rec., Furunkulose. \\
\hline 6. & M. E. & $11 / 2 \mathrm{~J}$. & 3,66 & 62 & Lues. (9.5. 139560 W.) \\
\hline 17.5 .13 & $2 \mathrm{~cm} \mathrm{H} \mathrm{H}_{2} \mathrm{O}$ & 一 & 4,04 & 一 & $\longrightarrow$ \\
\hline 7. & C. H. & $7 \mathrm{~J}$. & 7,14 & 90 & $\begin{array}{l}\text { Bronchialdrüsentbc., Asthenie. } \\
(21.5 .1310360 \text { W.) }\end{array}$ \\
\hline 8. & G. E. & $7 \mathrm{~J}$. & 5,82 & 87 & Pneumonie 1 Tag nach Krise. \\
\hline 9. & Fr. Ebr. & $4 \mathrm{~J}$. & 6,25 & 83 & Scharlach? rec. \\
\hline 10. & L. Sch. & $1 \mathrm{~J}$. & 5,87 & 73 & Scharlach? Varicellen rec. \\
\hline 11. & G. H. & $1 \mathrm{~J}$. & 5,72 & 92 & $\begin{array}{l}\text { Scharlach? Varicellen rec. } \\
(16.5 .137960 \text { W. })\end{array}$ \\
\hline 19. 5. 13 & $2 \mathrm{ccm} \mathrm{H} \mathrm{H}_{2} \mathrm{O}$ & - & 4,00 & - & - \\
\hline 12. & C. H. & $7 \mathrm{~J}$. & 7,49 & 95 & $\begin{array}{l}\text { leichte Bronchialdrüsentbc. } \\
(21.5 .1310360 \mathrm{~W} .)\end{array}$ \\
\hline 13. & H. Stg. & $5 \mathrm{~J}$. & 9,94 & 81 & schwere häm. Nephritis. Sepsis. \\
\hline 14. & G. R. & $12 \mathrm{~J}$. & 6,98 & 96 & leichte Epilepsie, gestern Absence. \\
\hline 15. & K. K. & $12 \mathrm{~J}$. & 7,00 & 107 & Chorea minor. \\
\hline 16. & R. R. & $10 \mathrm{~J}$. & 6,60 & 101 & Chorea minor. \\
\hline 17. & G. E. & $7 \mathrm{~J}$. & 5,96 & 93 & $\begin{array}{l}\text { Pneumonie rec. } 3 \text { Tage n. d. Krise. } \\
(15.5 .1312150 \text { W.) }\end{array}$ \\
\hline 24. 5. 13 & $2 \mathrm{cem} \mathrm{H}_{2} \mathrm{O}$ & 一 & 2,83 & 一 & - \\
\hline 18. & K. K. & $7 \mathrm{~J}$. & 4,87 & 96 & Asthma bronchiale. (5480 W.) \\
\hline 19. & C. $\mathrm{H}$. & $7 \mathrm{~J}$. & 5,86 & 100 & leichte Bronchialdrüsenschwellung \\
\hline 20. & A. B. & $7 \mathrm{~J}$. & 5,17 & 98 & Mikrosomie. \\
\hline 21 . & Gg. M. & $7 \mathrm{~J}$. & 5,11 & 90 & Meningealerschein. n. Parotitis. \\
\hline 22. & H. Stg. & $5 \mathrm{~J}$. & 7,30 & 77 & $\begin{array}{l}\text { hämorrhag. Nephritis, Sepsis. } \\
(20480 \text { W. })\end{array}$ \\
\hline 27. 5.13 & $2 \mathrm{ccm} \mathrm{H} \mathrm{H}_{2} \mathrm{O}$ & - & 3,54 & - & - \\
\hline 23. & E. Sp. & $6 \mathrm{Mt}$. & 5,03 & 88 & Ammenkind. \\
\hline 24. & L. N. & I J. & 5,85 & 100 & Pneumonia croup. 4 Tage v.d.Kris. \\
\hline 25. & M. E. & $11 / 2 \mathrm{~J}$. & 4,85 & 65 & Lues. \\
\hline 26. & A. D. & $2 W$. & 6,36 & 110 & $\begin{array}{l}\text { Meningoencephalocele, Nabeleite- } \\
\text { rung. (17.5.13 } 11280 \mathrm{~W} \text {.) }\end{array}$ \\
\hline 27. & K. U. & $2^{1} / 4 \mathrm{~J}$. & 4,76 & 44 & $\begin{array}{l}\text { Megalosplenie, Rachitis. } \\
(22.5 .138200 \text { W.) }\end{array}$ \\
\hline 17. 6.13 & $2 \mathrm{ccm} \mathrm{H} \mathrm{H}_{2} \mathrm{O}$ & - & 3,20 & - & - \\
\hline 28. & J. M. & $7 \mathrm{~J}$. & 4,52 & 90 & Bronchialdrüsentbc., Otitis geh. \\
\hline & B. L. & $10 \mathrm{~J}$. & 4,19 & 100 & $\begin{array}{l}\text { Bronchialdrüsentbc., Lymphatis- } \\
\text { mus. (26.6.13 6200 W.) }\end{array}$ \\
\hline $\begin{array}{l}30 . \\
31 .\end{array}$ & $\begin{array}{l}\text { F. Sch. } \\
\text { H. Stg. }\end{array}$ & $\begin{array}{ll}7 & \mathrm{~J} . \\
5 & \mathrm{~J}\end{array}$ & $\begin{array}{l}4,05 \\
5,65\end{array}$ & $\begin{array}{l}97 \\
63\end{array}$ & $\begin{array}{l}\text { Cystopyelitis, Albuminurie. } \\
\text { nach häm. Nephritis und Sepsis } \\
\text { Empyem und Pertussis. }\end{array}$ \\
\hline
\end{tabular}


tätigkeit des Blutes und von Gewebeflüssigkeiten im Kindesalter.

\begin{tabular}{|c|c|c|c|c|c|}
\hline Datum & Name & Alter & $\begin{array}{l}\text { Kataly- } \\
\text { satoren- } \\
\text { wert }\end{array}$ & $\mathbf{H b} \%$ & Diagnose \\
\hline 32. & A. A. & $10 \mathrm{~J}$. & 5,49 & 86 & $\begin{array}{l}\text { croup. Pneumonie, altes interlob. } \\
\text { Empyem } 6 \text { Tg. n. d. Krise. }\end{array}$ \\
\hline 33. & H. Schw. & $7 \mathrm{~J}$. & 4,35 & 93 & orthotische Albuminurie. \\
\hline 34. & H. W. & $2 \mathrm{~J}$. & 4,34 & 86 & Angina rec. \\
\hline 6. 13 & $2 \mathrm{com} \mathrm{H}_{2} \mathrm{O}$ & - & 3,52 & - & - \\
\hline 35. & E. Sp. & $61 / 2 \mathrm{Mt}$. & 4,19 & 90 & Ammenkind, ständigLeukocytose. \\
\hline 36. & A. Schm & $3 / 4 \mathrm{~J}$ & 4,51 & 62 & Lues. Intertrigo. \\
\hline 37. & M. F. & $3 / 4 \mathrm{~J}$ & 4,60 & 75 & Ekzem, exsudative Diathese. \\
\hline 38. & M. Fr. & $21 / 2 \mathrm{Mt}$. & 6,71 & 74 & ascendier.Cystopyelitis, Dyspepsie \\
\hline 39. & H. $\mathbf{H .}$ & 1 Mt. & 5,26 & 102 & Dyspepsie rec. \\
\hline 40. & Fr. M. & 2 Mt. & 4,95 & 90 & $\begin{array}{l}\text { Schwere Pyelitis. } \\
(21.6 .1312480 \text { W. })\end{array}$ \\
\hline 41. & E. R. & $3 / 4 \mathrm{~J}$ & 4,28 & 75 & Pneumonie rec. $4 \mathrm{Tg}$. $\mathrm{n}$. d. Krise. \\
\hline . 6.13 & $2 \mathrm{com} \mathrm{H}_{2} \mathrm{O}$ & - & 3,05 & 一 & - \\
\hline 42 & E. $\mathrm{F}$ & 9 J. & 5,08 & 100 & Bronchiektase \\
\hline 43 & M. $s$ & $7 \mathrm{~J}$. & 4,5 & 97 & Pneumonie rec. $8 \mathrm{Tg}$ \\
\hline 44 & & $5 \mathrm{~J}$. & 5 , & 92 & es Ödem (3.7.13 \\
\hline 45 & A. & $10 \mathrm{~J}$. & 5,15 & 96 & $\begin{array}{l}\text { Pneumonie rec., altes interlobäres } \\
\text { Empyem. }\end{array}$ \\
\hline 46. & L. Sch. & 3 J. & 5,41 & 98 & $\begin{array}{l}\text { Poliomyelitis rec. } \\
(10.7 .1310680 \text { W. })\end{array}$ \\
\hline 47. & H. F. & $4 \mathrm{~J}$. & 4,70 & 87 & Morbilli Stad. exanth. (3720 W.) \\
\hline
\end{tabular}

\begin{tabular}{|c|c|c|c|c|c|c|}
\hline Datum & Name & Alter & $\begin{array}{c}\text { Kataly- } \\
\text { satoren- } \\
\text { wert }\end{array}$ & $\mathbf{H b} \%$ & $\begin{array}{l}\text { Leuko- } \\
\text { cytenzahl }\end{array}$ & Diagnose \\
\hline .8 .13 & $2 \mathrm{ccm} \mathrm{H}_{2} \mathrm{O}$ & 一 & 4,00 & - & — & - \\
\hline 48. & H. Stg. & $5 \mathrm{~J}$. & 5,10 & 81 & 15400 & $\begin{array}{l}\text { nach Pneumokokken. } \\
\text { sepsis Empyem oper. }\end{array}$ \\
\hline 49. & H. $\mathbf{K}$. & $5 \mathrm{~J}$. & 4,43 & 71,5 & 10840 & Empyem operiert. \\
\hline 50. & Gg. $\mathbf{H}$. & $8 \mathrm{~J}$. & 4,81 & 105 & 11560 & Leichte Gastroenteritis. \\
\hline 21. 8. 13 & $2 \mathrm{ccm} \mathrm{H}_{2} \mathrm{O}$ & - & 1,70 & - & 一 & - \\
\hline 51 & E. E. & $11 \mathrm{~J}$. & 3,35 & 121 & 8032 & $\begin{array}{l}\text { Asthenie. Bronchial- } \\
\text { drüsentbc. }\end{array}$ \\
\hline 52. & I. L. & $\mathbf{1}^{1 / 2} \mathrm{~J}$ & 4,38 & 81 & 23920 & $\begin{array}{c}\text { chron. Pneumonie. Co- } \\
\text { ryza, Pertussis. }\end{array}$ \\
\hline 53. & A. W. & $3 \mathrm{~J}$. & 3,06 & 92,5 & 6400 & $\begin{array}{l}\text { akute Nierenschwellung } \\
\text { 1. Urämie. }\end{array}$ \\
\hline 23. 8.13 & $2 \mathrm{ccm} \mathrm{H}_{2} \mathrm{O}$ & - & 1,58 & 一 & - & 一 \\
\hline 54 & K Z & $13 \mathrm{~J}$. & 3,25 & 82,5 & 6040 & $\begin{array}{l}\text { Lues + Bronchial- } \\
\text { drüsentbc. }\end{array}$ \\
\hline 55. & B. L. & $10 \mathrm{~J}$. & 3,39 & 86,5 & 6880 & $\begin{array}{l}\text { Bronchialdrüsentbc. } \\
\text { Lymphatismus. }\end{array}$ \\
\hline 56. & B. Tr. & $3 \mathrm{~J}$. & 3,65 & 95,0 & 9800 & Masern rec. \\
\hline
\end{tabular}


E. Stettner: Untersuchungen über die Beeinflassung der Katalysatoren-

\begin{tabular}{|c|c|c|c|c|c|c|}
\hline Datum & Name & Alter & $\begin{array}{c}\text { Kataly- } \\
\text { satoren- } \\
\text { wert }\end{array}$ & $\mathrm{Hb}_{10}$ & $\begin{array}{c}\text { Leuko- } \\
\text { cytenzahl }\end{array}$ & Diagnose \\
\hline 57. & P. B. & $3^{1} / 2 \mathrm{~J}$. & 3,70 & 79,0 & 10600 & $\begin{array}{l}\text { Pneumonie rec, seit } 4 \\
\text { Wch. fieberfrei, nekro- } \\
\text { tisierende Wunde. }\end{array}$ \\
\hline 58. & K. V. & $10 \mathrm{~J}$. & 3,72 & 84,5 & 11300 & $\begin{array}{l}\text { Mitralinsufficienz u. Ste- } \\
\text { nose. Knötchenrheu- } \\
\text { matismus. }\end{array}$ \\
\hline 59. & L. H. & $11 \mathrm{~J}$. & 3,83 & 100 & 11520 & Angina rec. \\
\hline 25. 8. 13 & $2 \mathrm{~cm} \mathrm{H}_{2} \mathrm{O}$ & 一 & 1,92 & - & - & - \\
\hline 60. & O. Tr. & $6 \mathrm{~J}$. & 2,94 & 88 & 4640 & akutes Masernexanthem \\
\hline 61. & E. W. & $5 \mathrm{~J}$. & 3,52 & 90,5 & 6500 & Diphtherie. \\
\hline 62. & K. U. & $2^{1 / 2} \mathrm{~J}$ & 2,80 & 46 & 7480 & Megalosplenie, Rachitis. \\
\hline 63. & R. H. & $2 \mathrm{~J}$. & 3,18 & 84 & 10560 & nekrotisier. Gingivitis. \\
\hline 64. & J. B. & $9 \mathrm{~J}$. & 3,73 & 88 & 9400 & $\begin{array}{l}\text { Enuresis nocturna, } \\
\text { chron. Cystitis. }\end{array}$ \\
\hline 65. & W. St. & 8 Mt. & 3,50 & 84 & 11080 & $\begin{array}{l}\text { Impetiginöses Ekzem, } \\
\text { Furunkulose. }\end{array}$ \\
\hline 66. & B. Gn. & $3 \mathrm{~J}$. & 3,63 & 89,5 & 11480 & Masern rec. (5 Wch.). \\
\hline 67. & Ap. S. & $11 / 4 \mathrm{~J}$. & 3,41 & 89,0 & 14280 & Pertussis - Pneumonie. \\
\hline
\end{tabular}

Wie nach dem früher erwähnten Gesetze zu erwarten war, zeigen sich in unserer Aufstellung Schwankungen, welche dem Grade der Einwirkung von Eiweißspaltprodukten entsprechen. Für normale Fälle hätten wir nach den Weich ard tschen Voraussetzungen einen Parallelismus mit dem Hämoglobingehalt des Blutes erwarten können. Daß dieses nicht der Fall ist, beruht wohl darauf, daß mit dem Authenrieth - Königsbergerschen Apparat, mit welchem unser Hb-Gehalt bestimmt war, wie mit allen anderen gebräuchlichen Methoden lediglich die Färbekraft des Hämoglobins bestimmt werden kann, während dessen chemische Aktivität keineswegs zum Ausdruck kommt. Nachdem auf diesem Wege Korrelationen zwischen Hämoglobin und Blutkatalysator nicht festgestellt werden konnten, forschten wir nach Beziehungen zu anderen Blutbestandteilen. Schon die erste Versuchsreihe gab einen Hinweis, daß entzündliche Vorgänge die Katalysatorentätigkeit zu verändern vermochten, wir untersuchten daher in den letzten 20 Fällen, ob etwa zwischen dem Gehalt an Leukocyten und dem Verhalten des Katalysators Parallelen zu finden wären. In der Tat fand sich eine weitgehende Ubereinstimmung, indem einer Leukocytose eine Vermehrung der Blutkatalase, der Leukopenie eine Verminderung derselben entsprach. In den Versuchen am 23. VIII. 13 ging diese Ưbereinstimmung soweit, daß sich selbst kleine Differenzen im Leukocytengehalt in der Katalysatorenkurve widerspiegelten. Jedoch keine Regel ohne Ausnahme, dieses Wort hat vor allem bei biologischen Fragen Berechtigung. Die Ausnahmen kamen sofort am folgenden Versuchstag. Fall 61 
und 64 bieten einen zu hohen Katalysatorenwert, sie betreffen einen Fall von klinisch leichter Tonsillendiphtherie und einen Fall von Enuresis nocturna mit recht hartnäckiger Cystitis. Im Gegensatz zu diesen stehen die Fälle 62 und 67, ihr Wert ist geringer als erwartet wurde. Beim Falle 62 handelt es sich um einen hochgradigen Rachitiker mit starker Milzvergrößerung und erheblicher Verringerung der roten Blutkörperchen. Hier hat man wirklich den Eindruck, als ob der Mangel an Hämoglobingehalt den Katalysatorenwert herabsetze, oder sollte die Erythrocytenverminderung ausschlaggebend sein? Wenn dies der Fall wäre, wäre eine Erklärung für Fall 64 gefunden, denn dieses 9jährige Mädchen hat 5125000 Erythrocyten in $1 \mathrm{cmm}$, der alle anderen überragende Katalysatorenwert könnte demnach der Ausdruck einer Polycythämie sein. Aus alledem geht wohl nur soviel hervor, daß in vielen Fällen der Leukocytengehalt des Blutes für seine katalytische Fähigkeit maßgebend ist, daß aber auch der Hb-Gehalt und die Erythrocytenzahl berücksichtigt werden müssen. Keine von beiden Erklärungen eignen sich aber für Fall 67. Der gefundene Wert ist für den Leukocytengehalt von 14280 auffallend niedrig, das Blut stammt von einem Kinde mit sehr heftigem Keuchhusten und immer wiederkehrenden Bronchopneumonien. Am Untersuchungstag war das Kind fieberfrei, eine kurze Fieberperiode lag in den Tagen unmittelbar vorher. Vergleicht man diesen Wert mit anderen in postpneumonischen Tagen, so findet man in den Fällen $8,17,41,43$ immer wieder den niedrigen Katalysatorenwert wiederkehren, in Fällen, in welchen in der postpneumonischen Zeit Empyeme oder Inflammation von Resten eines früheren Empyems vorhanden ist, findet die Katalysatorenverminderung nicht statt (vgl. 31, $32,45,48)$. Hieraus ist zu schließen, daß noch allerlei Faktoren eine große Rolle spielen, die unabhängig vom Zellbestande des Blutes seine Katalysatoren energisch verändern können.

Die folgenden Untersuchungen sind der möglichsten Aufklärung dieser hypothetischen Verhältnisse zugeeignet. Nach Möglichkeit wurden die einzelnen Blutbestandteile, wie sie nach obengenannten Fragen in Betracht kommen, isoliert und ihre katalytische Fähigkeit in verschiedenen Verdünnungsgraden untersucht. Man gewinnt auf diese Weise recht anschauliche Kurven. Gleichzeitig wurden in weiteren Röhrchen die gleichen Verdünnungen auf einen Osmiumkatalysator einwirken lassen, da das eigentümliche Verhalten der Molke gegenüber dem kolloidalen Osmium, wie es die erste Kurve zeigt, unbedingt einer Nachforschung bedurfte.

Blut, Blutserum und Blutkatalysator der folgenden Versuchsreihen entstammen der Armvene eines gesunden 26 jährigen Mannes. Zunächst die Kurve der Verdünnungen des Gesamtblutes (Fig. 2). 
E. Stettner: Untersuchungen uber die Beeinflussung der Katalysatoren-

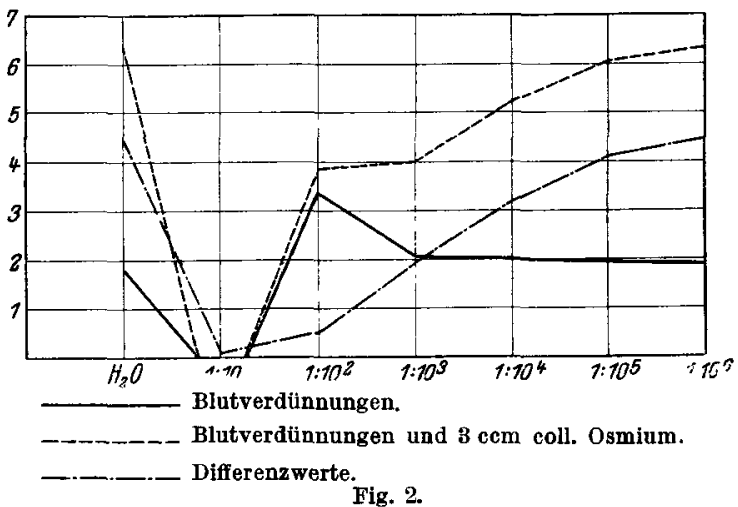

Die Kurve lehrt, daß Blut in der Verdünnung $1: 10$ mit frisch destilliertem Wasser keine katalytischen Fähigkeiten zeigt, im Gegenteil, die Bildung von Jodstärke völlig zu verhindern vermag, während das Kontrollkölbchen, in welchem sich an Stelle der Blutverdünnung $1 \mathrm{ccm} \mathrm{H}_{2} \mathrm{O}$ befand, nach $1 / 2$ Stunde bei der Titration den Wert von 1,91 gab. Dagegen wurde in der Verdünnung $1: 100$ gegenüber dem Wasserwert viel mehr Jodstärke gebildet, in dieser Verdünnung wirkte das Blut also katalytisch, in stärkeren Verdünnungen wurde diese Fähigkeit langsam wieder verloren. Die gestrichelte Linie zeigt das Verhalten im Verein mit $3 \mathrm{ccm}$ Osmium, durch Subtraktion der erst gefundenen Werte von diesen gewinnt man Werte, die ein Bild von der Beeinflussung des kolloidalen Osmiums durch das Blut entwerfen; hier fällt auf, daß in der stärksten Konzentration der Katalysator völlig vergiftet wird, d. h. nicht mehr imstande ist, die Bildung von Jodstärke zu vermehren, selbst in der Verdünnung $1: 100$, in welcher das Blut allein schon katalytisch wirkt, wird das kolloidale Osmium noch stark in seiner Wirkung gehemmt, in stärkeren Verdünnungen verlieren sich die vergiftenden Eigenschaften des Blutes.

Kurven des Blutserums (Fig. 3):

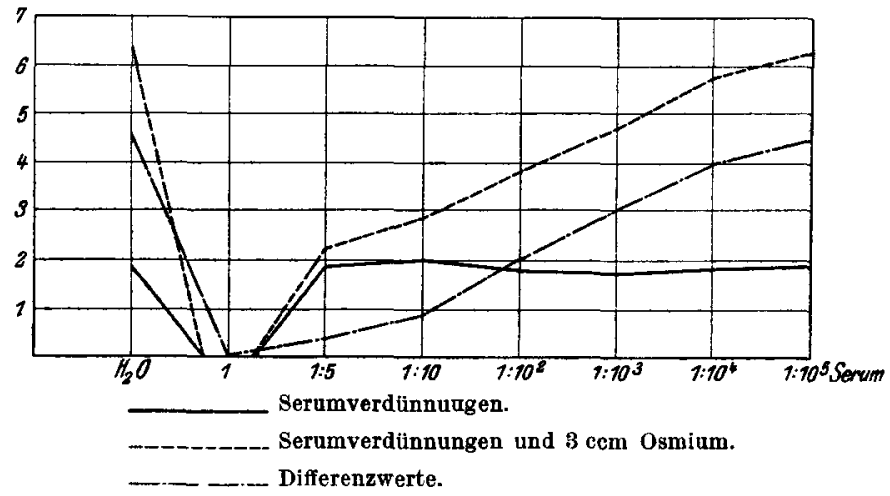

Fig. 3. 
Die Kurven sind in analoger Weise gewonnen. Die Serumkurve verläuft wesentlich flacher wie die Blutkurve, von einer katalytischen Fähigkeit sind in der Verdünnung 1: 100 nur Spuren zu bemerken. In stärkster Konzentration, in diesem Fall als unverdünntes Blutserum, vermag es ganz analog dem Blutkomplex die Jodstärkebildung völlig zu verhindern. Die Einwirkung auf das kolloidale Osmium geschieht wiederum im Sinne der Lähmung dieses Katalysators.

Interessant ist auch das Verhalten eines sterilen Pleuraexsudats und einer Ascitesflüssigkeit; beide Serumabkömmlinge wurden nur mit Osmium zusammen untersucht. (Fig. 4.)

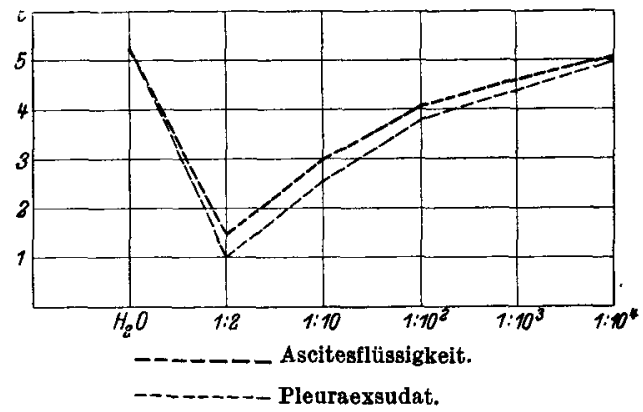

Fig. 4.

Der weitgehende Parallelismus beider Kurven untereinander sowie mit der obenangeführten Serumosmiumkurve ist so auffallend, daß ohne weiteres die Verwandtschaft der drei Flüssigkeiten zu erkennen ist.

Wie verhält sich nun der nach Weichardt dargestellte Blutkatalysator des gleichen Blutes? Das Trockenblut war zu $250 \mathrm{mg}$ abgewogen und in $25 \mathrm{ccm} \mathrm{H}_{2} \mathrm{O}$ gelöst; damit in dieser hohen Konzentration eine vollkommene Lösung erreicht wurde, wurden noch 3 Tropfen $1 / 10 \mathrm{n}$ $\mathrm{NaOH}$ hinzugesetzt. Von dieser Stammlösung ausgehend wurden dann die weiteren Verdünnungen hergestellt. Die Verdünnung $1: 5$ entspricht der sonst gebräuchlichen Weichardtschen Konzentration.

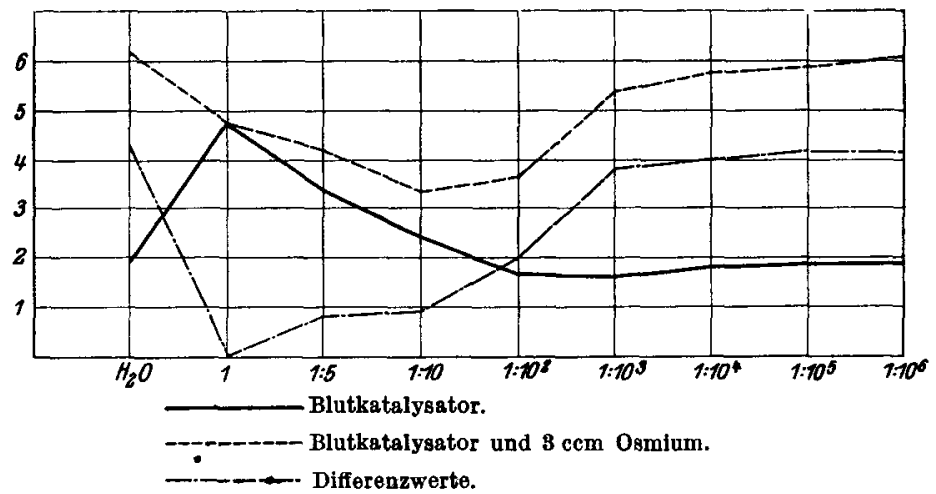

Fłg. $\overline{0}$. 
Die Kurve des Blutkatalysators (Fig. 5) zeigt nun ein ganz anderes Verhalten als die vorhergehenden. Hier ist bereits in der stärksten Konzentration eine mächtige Anregung des Prozesses zu verzeichnen, die allmählich mit dem höheren Verdünnungsgrade abnimmt. Die Einwirkung auf das kolloidale Osmium ist aber, ganz analog den Erfahrungen beim Gesamtblut und beim Blutserum, wiederum eine starke Lähmung der Katalase. Vergegenwärtigen wir uns den Hergang der Blutkatalysatorbereitung, so müssen wir annehmen, daß wir in ihm einen Körper vor uns haben, in welchem die wasserlöslichen Bestandteile der roten und weißen Blutkörperehen vorhanden sind, während die Serumbestandteile und alle diejenigen Körper entfernt sind, die durch die isotonische NaCl-Lösung weggewaschen werden können. Man gewinnt auf diese Weise vorzüglich wohl den roten Blutfarbstoff, aber auch alle wasserlöslichen Innenkörper der roten und weißen Blutkörperchen. Die Gesamtheit dieser Körper besitzen die für unsere Versuche maßgebenden Eigenschaften, enthalten die Katalysatoren, während deren Tätigkeit in hohen Konzentrationen des Blutkomplexes durch die Anwesenheit von Serum verdeckt wird.

Es könnte noch die Frage aufgeworfen werden, wie es kommt, daß unser Osmiumkatalysator stets in so entschiedener Weise gelähmt wird. Dieses erhellt daraus, daß bei anorganischen Katalysatoren, im Gegensatz zum organischen Blutkatalysator bei Einwirkung von Eiweißspaltprodukten nur ein ganz kurzes Anregungsstadium zustande kommt, so daß es nur bei Anwendung feiner abgestufter Lösungen beobachtet werden kann; dementsprechend sehen wir in unseren Versuchen nur Lähmung. Vielleicht kommt das Anregungsstadium auch deshalb nicht zur Darstellung, weil es sich bei vorliegenden Versuchen stets um die Wirkung zweier Katalysatoren handelt, die sich gegenseitig beeinflussen. Die folgende Untersuchung soll zeigen, welcher der beiden Katalysatoren den anderen mit stärkerer Kraft in seiner Wirksamkeit zu verändern vermag. Diesmal haben wir die Verhältnisse umgekehrt, indem wir den Blutkatalysator in konstanter Größe verwandten, während wir das kolloidale Osmium abgestuft verdünnten. Durch einen Vergleich der vorhergehenden Kurve mit dieser (Fig. 6) gewinnt man einigen Einblick in den Mechanismus der gegenseitigen Einwirkung.

Der fast völlig gerade Verlauf der gestrichelten Kurve sagt mit aller Deutlichkeit, wie wenig der Blutkatalysator durch das kolloidale Osmium tangiert wird. $1 \mathrm{ccm}$ Osmium $+1 \mathrm{ccm}$ Blutkatalysator $0,1: 50,0$ vermögen die Jodstärkebildung zu vermehren, aber nicht in so hohem Maße, daß sie dem Additionswert entsprächen, der aus der Wirkung des Blutkatalysators und des kolloidalen Osmiums jedes für sich allein gewonnen würde. In der Differenzkurve kommt wiederum deutlich die gegenseitige Lähmung der Katalysatoren zum Ausdruck. Ihr Verlauf 
weicht aber von den früheren in dem Sinne ab, daß schon bei der Verdünnung 1 : 10 der Wasserdifferenzwert wieder erreicht ist, während dies bei allen anderen Kurven erst viel später der Fall ist. Die Kurve lehrt also, daß der organische Blutkatalysator viel geringer von dem anorganischen kolloidalen Osmium verändertwird, wie umgekehrt. Es könnte noch der Einwand erhoben werden, daß es sich gar nicht um die gegenseitige Beeinflussung zweier Katalysatoren handelt, sondern daß die Eiweißspaltprodukte, die im Blutkatalysator vorhanden sein können, auf das kolloidale Osmium derart lähmend einwirken. Dem kann aber nicht so sein, denn, wie die letzte Kurve zeigt, verläuft die Linie, welche die Werte der Wirkungsweise für Blutkatalysator + kolloidales Osmium angibt, nahezu völlig geradlinig, während doch sonst bei dem zunehmenden Mißverhältnis von Eiweißspaltprodukten zum Osmiumkatalysator eine Senkung der Kurve an irgendeiner Stelle stattfinden müßte.

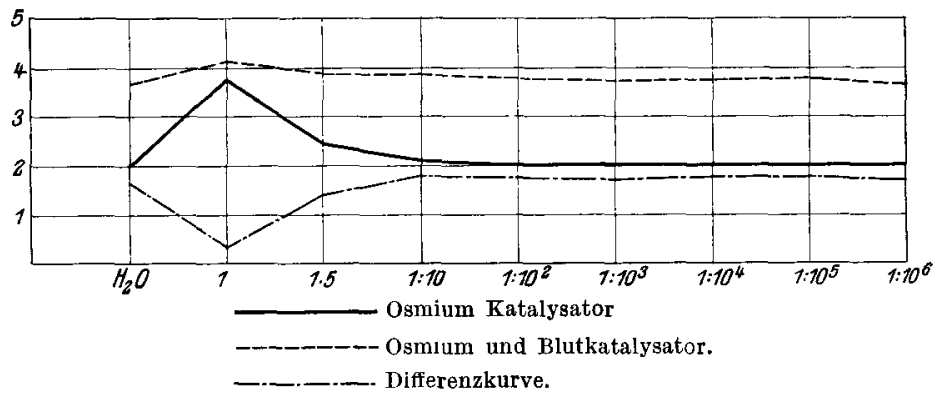

Fig. 6.

Zur Frage der Beeinflussung zweier Katalysatoren wäre zusammenfassend zu bemerken, daß beide sich lähmend beeinflussen; dies gilt für den Molken- und die Blutkatalysatoren gegenüber dem kolloidalen Osmium. Die Wirkung auf das kolloidale Osmium ist eine viel intensivere, während die beiden geprüften organischen Katalysatoren dem anorganischen gegenüber sich als widerstandsfähiger erwiesen haben.

Anhangsweise sei noch eine Untersuchung eines sehr pneumokokkenreichen Empyemeiters auf seine katalytischen Fähigkeiten mitgeteilt.

Die Kurve (Fig. 7) ist aus Mangel an Material leider unvollständig, jedoch kommen die außerordentlichen katalytischen Eigenschaften des Eiters in deutlichster Weise zum Ausdruck. In der Verdünnung 1:1 nehmen diese Eigenschaften noch zu; in dieser Verdünnung vermögen die in großer Menge vorhandenen Eiweißspaltprodukte offenbar den Eiterkatalysator anzuregen. Sehr interessant verläuft die Osmiumkurve, die Eiweißspaltprodukte vermochten hier den Katalysator bis in hohe Ver- 
dünnungen entschieden zu lähmen. Die Widerstandsfähigkeit eines organischen Katalysators gegenüber Eiweißspaltprodukten ist hier im Vergleich zum anorganischen deutlich größer festzustellen.

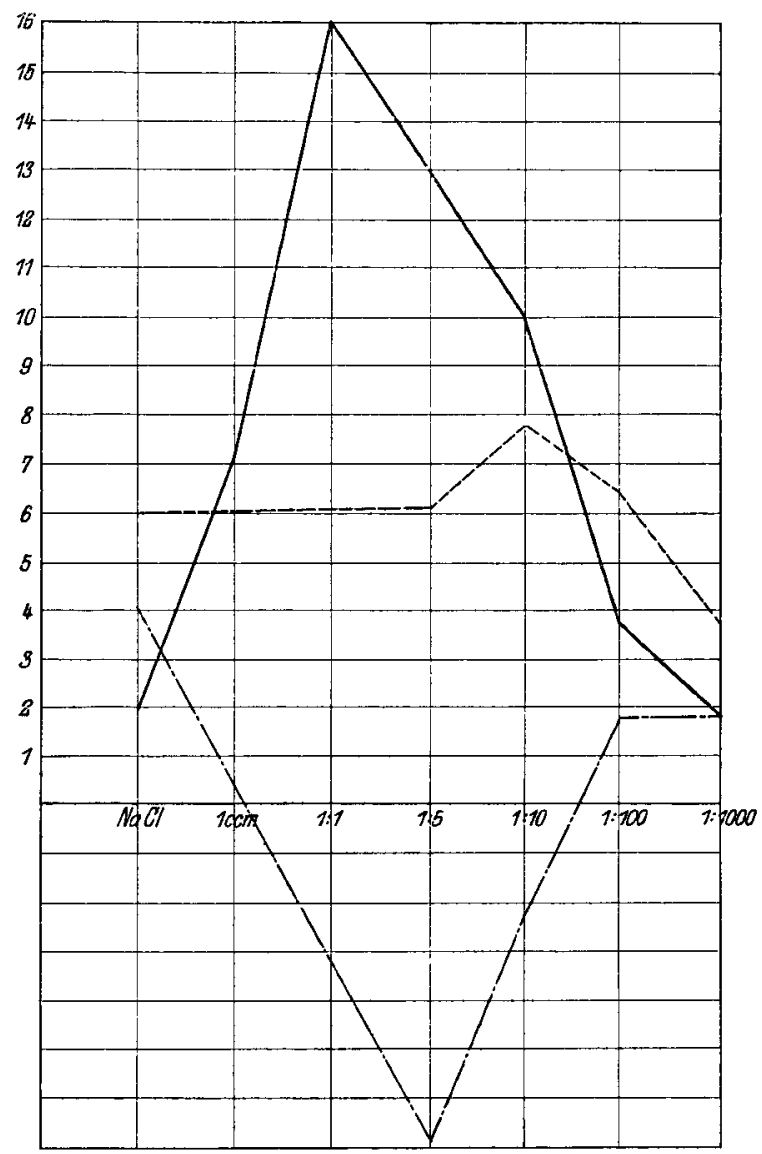

Fig. 7.

Für die Zukunft ergeben sich eine Reihe interessanter Fragestellungen, die mit Hilfe der Weichardtschen Methode lösbar sind. Bei Berïcksichtigung des Gesetzes über die Beeinflußbarkeit der Katalysatoren durch Eiweißspaltprodukte erscheinen beispielsweise Versuche aussichtsreich, Alter und Eigenschaften verschiedener Eitersorten zu bestimmen, da mit zunehmendem Alter und mit der Vermehrung an verdauenden Eigenschaften sich Eiweißspaltprodukte in reichlicher Menge antreffen lassen. Diese sind nun mit Hilfe unserer quantitativen Methode in bester Weise bestimmbar. Vielleicht ergeben sich Unterschiede je nach der Art der Mikroorganismen, welche die Eiterbildung 
provoziert haben, oder auch Anhaltspunkte in der Virulenzfrage der pathogenen Mikroorganismen ähnlich den Ergebnissen, wie sie Rosenthal und Ba mberger gefunden haben. Aussichten in der Diagnosen-, vielleicht auch in der Prognosenstellung werden sich der Beantwortung dieser Fragen anschließen. Therapeutische Ausblicke scheinen nicht ohne weiteres gegeben zu sein; aus vorliegenden Versuchen geht nur so viel hervor, daß kolloidale Metalle in der Weise wie sie bei Infektionserkrankungen angewendet werden, wenn sie wirksam sind, in ihren katalysatorischen Eigenschaften nur dann in Betracht kommen, wenn sie in viel höheren Dosen angewandt werden. Durch den Nachweis der Überlegenheit organischer Katalysatoren über die anorganischen wäre in Zukunft unsere Aufmerksamkeit auf organische Katalysatoren zu richten und deren therapeutische Wirksamkeit zu prüfen.

\section{Schlußsätze.}

1. Die Katalysatoreigenschaften des Blutes stehen in Beziehung zu seinem Zellbestande und seinem Hämoglobingehalte. Vielfach hebt sich die katalytische Kraft bei Infektionserkrankungen gleichzeitig mit dem Auftreten einer Leukocytose.

2. Der nach Weichard t dargestellte Blutkatalysator zeigt die katalytischen Fähigkeiten am reinsten.

3. Dem Blutserum kommen in stärkerem Maße katalysator hemmende Eigenschaften zu.

4. Bei Anwendung eines anorganischen Osmiumkatalysators zeigt sich eine gegenseitige Beeinflussung mit dem Blutkatalysator im Sinne der Lähmung. Der organische Blutkatalysator erweist sich gegenüber der Einwirkung eines anorganischen Katalysators (kolloidales Osmium) und gegenüber der Einwirkung von Eiweißspaltprodukten widerstandsfähiger als kolloidales Osmium.

5. Die mit dem Weichard tschen Blutkatalysator angestellten Versuche ergeben in drei Fällen für das Spasmophilieblut eine Verminderung der katalytischen Fähigkeiten.

Versuch mit Kuhmilchmolke (Fig. 1).

\begin{tabular}{|c|c|c|c|c|c|c|}
\hline $\begin{array}{l}3 \mathrm{ccm} \mathrm{H}_{2} \mathrm{O} \\
2 \mathrm{ccm} \mathrm{H}_{2} \mathrm{O}\end{array}$ & $\begin{array}{c}== \\
2 \mathrm{ccm} \text { Molke } \\
1: 1\end{array}$ & $\begin{array}{c}= \\
= \\
1: 10\end{array}$ & $\begin{array}{c}= \\
= \\
1: 10^{2}\end{array}$ & $\begin{array}{c}= \\
= \\
1: 10^{3}\end{array}$ & $\begin{array}{c}= \\
= \\
1: 10^{4}\end{array}$ & $\begin{array}{c}= \\
= \\
1: 10^{5}\end{array}$ \\
\hline 1,92 & 10,0 & 13,45 & 3,78 & 1,5 & 1,50 & 1,68 \\
\hline $\begin{array}{l}3 \mathrm{ccm} \text { Osmiun } \\
2 \mathrm{ccm} \mathrm{H}_{2} \mathrm{O}\end{array}$ & $\begin{array}{c}= \\
2 \mathrm{ccm} \text { Molke } \\
1: 1\end{array}$ & $\begin{array}{c}= \\
= \\
1: 10\end{array}$ & $\begin{array}{c}= \\
= \\
1: 10^{2}\end{array}$ & $\begin{array}{c}= \\
= \\
1: 10^{3}\end{array}$ & $\begin{array}{c}= \\
= \\
1: 10^{4}\end{array}$ & $\begin{array}{c}= \\
= \\
1: 10^{5}\end{array}$ \\
\hline \multicolumn{6}{|c|}{ Differenz. } & 5,43 \\
\hline$+4,28$ & $+0,45$ & $-0,20$ & $+0,20$ & $+3,63$ & $+3,89$ & $+3,75$ \\
\hline
\end{tabular}


234 E. Stettner: Untersuchungen über die Beeinflussung der Katalysatoren-

Versuch mit Blutverdünnungen (Fig. 2).

\begin{tabular}{c|c|c|c|c|c|c}
\hline \hline $3 \mathrm{ccm} \mathrm{H}_{2} \mathrm{O}$ & $\begin{array}{c}= \\
1 \mathrm{ccm} \text { Blut } \\
1: 10\end{array}$ & $\begin{array}{c}= \\
=\end{array}$ & $\begin{array}{c}= \\
=\end{array}$ & $\begin{array}{c}= \\
=\end{array}$ & $\begin{array}{c}= \\
=\end{array}$ & $\begin{array}{c}= \\
= \\
1 \mathrm{ccm} \mathrm{H}_{2} \mathrm{O}\end{array}$ \\
\hline 1,91 & 0 & 3,39 & 2,04 & 2,03 & 1,95 & 1,96 \\
\hline $3 \mathrm{ccm}$ Osmium & $=$ & $=$ & $=$ & $=$ & $=$ & $=$ \\
& $1 \mathrm{ccm} \mathrm{Blut}$ & $=$ & $=$ & $=$ & $=$ & $=$ \\
$1 \mathrm{ccm} \mathrm{H}_{2} \mathrm{O}$ & $1: 10$ & $1: 10^{2}$ & $1: 10^{3}$ & $1: 10^{4}$ & $1: 10^{5}$ & $1: 10^{6}$ \\
\hline 6,28 & 0 & 3,83 & 3,98 & 5,23 & 6,03 & 6,35
\end{tabular}

Differenz.

$+4,37 \quad$ । $0 \quad|+0,44|+1,94+3,20+4,08 \mid+4,45$

Versuch mit Blutserum (Fig. 3).

\begin{tabular}{|c|c|c|c|c|c|c|c|}
\hline $\begin{array}{l}3 \mathrm{ccm} \mathrm{H}_{2} \mathrm{O} \\
1 \mathrm{~cm} \mathrm{H}_{2} \mathrm{O}\end{array}$ & $\begin{array}{c}= \\
1 \mathrm{ccm} \text { Serum }\end{array}$ & $\begin{array}{c}= \\
= \\
1: 5\end{array}$ & $\begin{array}{c}== \\
= \\
1: 10\end{array}$ & $\begin{array}{c}= \\
= \\
1: 10^{2}\end{array}$ & $\begin{array}{c}= \\
= \\
1: 10^{3} \\
\end{array}$ & $\begin{array}{c}= \\
= \\
1: 10^{4}\end{array}$ & $\begin{array}{c}= \\
= \\
1: 10^{5}\end{array}$ \\
\hline 1,87 & 0 & 1,88 & 2,03 & 1,83 & 1,75 & 1,86 & 1,88 \\
\hline $\begin{array}{l}\text { ccm Osmium } \\
1 \mathrm{ccm} \mathrm{H}_{2} \mathrm{O}\end{array}$ & $\begin{array}{c}= \\
1 \mathrm{ccm} \text { Serum }\end{array}$ & $\begin{array}{l}= \\
= \\
1: 5\end{array}$ & $\begin{array}{c}= \\
= \\
1: 10\end{array}$ & $\begin{array}{c}= \\
= \\
1: 10^{2}\end{array}$ & $\begin{array}{c}= \\
= \\
1: 10^{3} \\
\end{array}$ & $\begin{array}{c}= \\
= \\
1: 10^{+}\end{array}$ & $\begin{array}{c}= \\
= \\
1: 10^{3}\end{array}$ \\
\hline \multicolumn{8}{|c|}{ Differenz. } \\
\hline$+4,47$ & 0 & 0,3 & $+0,8$ & 2,0 & +2, & $+3,99$ & $+4,4$ \\
\hline
\end{tabular}

Versuch mit Pleuraexsudat und Ascitesflüssigkeit (Fig. 4).

\begin{tabular}{|c|c|c|c|c|c|}
\hline $\begin{array}{c}3 \mathrm{ccm} \text { Osmium } \\
1 \mathrm{ccm} \mathrm{H}_{2} \mathrm{O} \\
\end{array}$ & 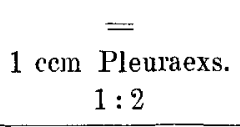 & $\begin{array}{c}= \\
= \\
1: 10\end{array}$ & $\begin{array}{c}= \\
= \\
1: 10^{2}\end{array}$ & $\begin{array}{c}= \\
= \\
1: 10^{3}\end{array}$ & $\begin{array}{c}= \\
= \\
1: 10^{4}\end{array}$ \\
\hline 5,22 & 1,04 & 2,62 & 3,86 & 4,43 & 4,99 \\
\hline $\begin{array}{l}3 \text { ccm Osmium } \\
1 \mathrm{ccm} \mathrm{H}_{2} \mathrm{O}\end{array}$ & $\begin{array}{c}= \\
1 \mathrm{ccm} \text { Ascitesfl. } \\
1: 2\end{array}$ & $\begin{array}{l}= \\
= \\
1: 10\end{array}$ & $\begin{array}{c}= \\
= \\
1: 10^{2}\end{array}$ & $\begin{array}{c}= \\
= \\
1: 10^{3}\end{array}$ & $\begin{array}{c}= \\
= \\
1: 10^{4}\end{array}$ \\
\hline 5,22 & 1,44 & 2,95 & 4,08 & 4,65 & 5,10 \\
\hline
\end{tabular}


tätigkeit des Blutes und von Gewebeflüssigkeiten im Kindesalter.

Versuch mit Blutkatalysator (Fig. 5).

\begin{tabular}{|c|c|c|c|c|c|c|c|c|}
\hline $\begin{array}{l}3 \mathrm{ccm} \mathrm{H}_{2} \mathrm{O} \\
1 \mathrm{ccm} \mathrm{H}_{2} \mathrm{O}\end{array}$ & $\begin{array}{c}= \\
1 \mathrm{ccm} \text { B.K. }\end{array}$ & $\begin{array}{c}= \\
= \\
1: 5\end{array}$ & $\begin{array}{c}= \\
= \\
1: 10\end{array}$ & $\begin{array}{c}= \\
= \\
1: 10^{2}\end{array}$ & $\begin{array}{c}= \\
= \\
1: 10^{3}\end{array}$ & $\begin{array}{c}= \\
= \\
1: 10^{4}\end{array}$ & $\begin{array}{c}= \\
= \\
1: 10^{5}\end{array}$ & $\begin{array}{c}= \\
= \\
1: 10^{6}\end{array}$ \\
\hline 1,90 & 4,78 & 3,4 & 100 & 1,67 & 1,59 & 1,80 & 1,04 & 1,86 \\
\hline $\begin{array}{l}\text { ccm Osmium } \\
1 \mathrm{ccm} \mathrm{H}_{2} \mathrm{O}\end{array}$ & $\begin{array}{c}= \\
1 \mathrm{ccm} \mathrm{B.K.}\end{array}$ & $\begin{array}{c}= \\
= \\
1: 5\end{array}$ & $\begin{array}{c}= \\
= \\
1: 10\end{array}$ & $\left\{\begin{array}{c}= \\
= \\
1: 10^{2}\end{array}\right.$ & $\begin{array}{c}= \\
= \\
1: 10^{3}\end{array}$ & $\begin{array}{c}= \\
= \\
1: 10^{4}\end{array}$ & $\begin{array}{c}= \\
= \\
1: 10^{5}\end{array}$ & $\begin{array}{c}= \\
= \\
1: 10\end{array}$ \\
\hline 6,20 & 4,79 & 4,21 & 3,34 & 3,65 & 5,38 & 5,81 & 5,90 & 6,02 \\
\hline \multicolumn{9}{|c|}{ ffferenz. } \\
\hline
\end{tabular}

Umkehrungsversuch mit Blut- und Osmiumkatalysator (Fig. 6).

\begin{tabular}{|c|c|c|c|c|c|c|c|c|c|}
\hline $\begin{array}{c}1 \mathrm{ccm} \mathrm{H}_{2} \mathrm{O} \\
3 \mathrm{ccm} \text { Osmium }\end{array}$ & $\begin{array}{l}1 \mathrm{ccm} \mathrm{H}_{2} \mathrm{O} \\
1 \mathrm{ccm} \mathrm{H}_{2} \mathrm{O}\end{array}$ & $\begin{array}{c}= \\
1 \mathrm{ccm} \text { Osmium }\end{array}$ & $\begin{array}{c}= \\
= \\
1: 5\end{array}$ & $\begin{array}{c}= \\
= \\
1: 10\end{array}$ & $\begin{array}{c}= \\
= \\
1: 10^{2}\end{array}$ & $\begin{array}{c}= \\
= \\
1: 10^{3}\end{array}$ & $\begin{array}{c}= \\
= \\
1: 10^{4}\end{array}$ & $\left\{\begin{array}{c}= \\
= \\
1: 10^{5}\end{array}\right.$ & $\left\{\begin{array}{c}= \\
= \\
1: 10^{6}\end{array}\right.$ \\
\hline 5,89 & 2,00 & 3,77 & 2,46 & 2,08 & 2,04 & 2,0 & 2,00 & 2,02 & 2,01 \\
\hline $\begin{array}{c}1 \mathrm{ccm} \text { B.K. } \\
3 \mathrm{ccm} \text { Osmium }\end{array}$ & 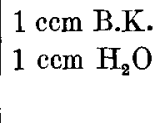 & $=$ & $\begin{array}{c}= \\
= \\
1: 5\end{array}$ & $\begin{array}{c}= \\
= \\
1: 10\end{array}$ & $\begin{array}{c}= \\
= \\
1: 10^{2}\end{array}$ & $\begin{array}{c}= \\
= \\
1: 10^{3}\end{array}$ & $\begin{array}{c}= \\
= \\
1: 10^{4}\end{array}$ & $\begin{array}{c}= \\
= \\
1: 10^{5}\end{array}$ & $\begin{array}{c}= \\
= \\
1: 10^{6}\end{array}$ \\
\hline 4,28 & 3,67 & 4,1 & 3,87 & 3,86 & 3,82 & 3,76 & 3,78 & 3,82 & 3,7 \\
\hline
\end{tabular}

Eiterversuch (Fig. 7).

\begin{tabular}{c|c|c|c|c|c|c}
\hline \hline $1 \mathrm{ccm} 0,9 \% \mathrm{NaCl}$ & $1 \mathrm{ccm}$ Eiter & $\begin{array}{c}= \\
1: 1\end{array}$ & $\begin{array}{c}= \\
1: 5\end{array}$ & $\begin{array}{c}= \\
1: 10\end{array}$ & $\begin{array}{c}= \\
1: 10^{2}\end{array}$ & $\begin{array}{c}= \\
1: 10^{3}\end{array}$ \\
\hline 1,89 & 7,1 & $>10,0$ & wenig $>10,0$ & 10,0 & 3,62 & 1,87 \\
\hline $3 \mathrm{ccm}$ Osmium & $=$ & $=$ & $=$ & $=$ & $=$ & $=$ \\
$1 \mathrm{ccm} 0,9 \mathrm{NaCl}$ & $1 \mathrm{ccm}$ Eiter & $=$ & $=$ & $=$ & $=$ & $=$ \\
& & $1: 1$ & $1: 5$ & $1: 10$ & $1: 10^{2}$ & $1: 10^{3}$ \\
\hline 6,0 & - & - & 6,12 & 7,75 & 5,40 & 3,65
\end{tabular}

Differenz.

$+4,11|-|-\mid-$ ca. $6,90|-2,25|+1,78 \mid+1,78$ 
236 E. Stettner: Untersuchung. ủb. d. Beeinflussung d. Katalysatorentatigkeit usw.

\section{Literaturverzeichnis.}

1. W. Weichardt, Über Ausatemluft. Archiv f. Hyg. 65, 252.

2. - u. H. Stötter, Über verbrauchte Luft. Archiv f. Hyg. 75, 265.

3. - u. C. Kelber, Über Luftuntersuchungen. Münch. med. Wochenschr. 1912, Nr. 35.

4. - u. Müller, Zentralbl. f. d. ges. Physiol. u. Pathol. d. Stoffw. 1911, Nr. 9.

5. -- Über neue chemische Methoden und ihre Verwertung. Sitzung d. Gesellsch. f. Morphol. u. Physiol. München, 21. Mai 1912.

6. - Untersuchungen über die Beeinflussung von Katalysatoren durch Eiweißspaltprodukte. Sitzungsber. d. physik.-med. Soz. Erlangen 44. 1912.

7. - Über Ermüdungsstoffe. Stuttgart, Ferd. Enke 1912 und Handb. d. pathogenen Mikroorganismen von Kolle-Wassermann, 2. Aufl., Bd. II.

8. - u. Schwenk, Über Beeinflussung von Katalysatoren durch Eiweißspaltprodukte. Zentralbl. f. Bakt. u. Parasitenk., I. Abt. Orig. 6z, 384. 1912.

9. - - Über verbrauchte Luft. Zeitschr. f. experim. Med. 1, 282. 1913.

10. - u. H. Schlee, Über das Studium unbekannter Gemische mit Hilfe von Katalysatoren. Zeitschr. f. d. ges. experim. Med. 1, 472.1913.

11. E. Engelhorn, Über die Beeinflussung des Hämoglobinkatalysators in der Schwangerschaft. (Weichardtsche Reaktion.) Münch. med. Wochenschrift 1913, Nr. 22.

12. E. Freudenberg u. L. Klocmann, Untersuchungen zum Spasmophilieproblem. Jahrb. f. Kinderheilk. 28, 47. 1913.

13. E. Rosenthal u. L. Bamberger, Experimentelle Untersuchungen über die Beeinflussung der Platinkatalyse durch Bakterienfiltrate. Zeitschr. f. Immunitätsf. u. experim. Ther. 19, Heft 1. 1913. 